\title{
Constrained Blind Source Separation by Morphological Characteristics and its Application in Modal Analysis
}

\author{
Teng Gong ${ }^{1}$, Zhousuo Zhang ${ }^{2}$, and Huan Wang ${ }^{3}$ \\ ${ }^{1,2,3}$ State Key Laboratory for Manufacturing Systems Engineering, Xi'an Jiaotong University, Xi'an, Shaanxi, 710049, PR \\ China \\ gong.teng@stu.xjtu.edu.cn \\ zzs@xjtu.edu.cn \\ wanghuan0890@stu.xjtu.edu.cn
}

\begin{abstract}
Semi-blind source separation algorithm is widely concerned for its advantages over classical blind source separation algorithm. However, in practical applications, it is often a difficult problem to design reference signals, which should be closely related to the desired source signals. Therefore the algorithm of constrained blind source separation by morphological characteristics is proposed in this paper, including three steps: the establishment of the enhanced contrast function, the optimization calculation and the extraction of multiple source signals. Firstly, the indexes measuring the morphological characteristics of a source signal are constructed based on the known prior information and introduced into the traditional contrast function to establish an enhanced contrast function, extending the use of prior information. Then, the optimization calculation is accomplished by genetic algorithm, obtaining a single source signal. Finally, the extraction of multiple source signals is realized by cluster analysis. The proposed algorithm is applied to the modal analysis under random excitation. The spectrum symmetry index is constructed and introduced into the kurtosis contrast function to establish the enhanced contrast function, thus realizing the extraction of each signal modal response. The extraction results show the effectiveness and superiority of the algorithm.
\end{abstract}

\section{INTRODUCTION}

The blind source separation (BSS) method proposed at the end of the twentieth century can separate and extract each source signal from mixed observation signals without or only a small amount of prior knowledge. In recent years, BSS technology has been widely used in the field of engineering. Some progress has been made in structural

Teng Gong et al. This is an open-access article distributed under the terms of the Creative Commons Attribution 3.0 United States License, which permits unrestricted use, distribution, and reproduction in any medium, provided the original author and source are credited. vibration signal analysis (He, Chen, Zhang, Sun \& Hu, 2018), structural damage identification (Sadhu, Narasimhan \& Antoni, 2017) and modal analysis (Chang, Liu, Hu \& Nagarajaiah, 2016). In the field of Prognostics and Health Management (PHM), the application of blind source separation technology has also achieved remarkable effects. Benkedjouh, Zerhouni, and Rechak (2018) proposed a new intelligent method for tool wear condition monitoring based on continuous wavelet transform (CWT) and BSS techniques. Zhang, Gao, Liu, Farzadpour, Grebe, and Tian (2017) propose an adaptive parameter BSS approach based on the adaptive time-frequency distributions theory in order to deal with the non-stationary blind separation problem and apply it to wheel defect monitoring.

Constrained BSS (Zhao, Kong, Jiang \& Qu, 2014), also known as semi-BSS, has received extensive attention because of the improvement of the accuracy of separation by exploiting the prior information, and has made significant progress (Zhang, Zhang, Cheng, Zhu \& He, 2015). In the constrained BSS algorithm, the reference signal similar to the source signal is constructed by using the prior information of the source signal, and it is introduced into the algorithm in the form of additional constraints. In practical applications, the construction of reference signals is a difficult problem. On the one hand, the construction of reference signals has higher requirements for prior knowledge so as to construct complete sequences of reference signals. On the other hand, the inaccuracy of the reference signals will have an adverse effect on the extraction of source signals. These greatly limit the application of reference signals. The known priori information is often less, so it is necessary to use a small amount of prior information to improve the separation performance. Therefore, this paper proposes constrained blind source separation by morphological characteristics. The morphological characteristics indexes are constructed according to a small amount of prior information and then introduced into the blind source separation algorithm as a 
constraint to enhance the separation performance of the algorithm.

The application of blind source separation in modal analysis has made some achievements. However, there are few applications of BSS in structural modal analysis under random excitation. In this paper, the algorithm is applied to modal analysis under random excitation to verify the effectiveness and superiority of the proposed algorithm.

\section{CONSTRAINED BLind SOURCE SEParation by MORPHOLOGICAL CHARACTERISTICS}

\subsection{BSS Model}

The linear instantaneous BSS model could be described as follows:

$$
x_{i}=\sum_{i=1}^{n} a_{i j} s_{j} \quad i=1,2, \cdots, m, j=1,2, \cdots n
$$

It can also be written in the form of a matrix:

$$
\boldsymbol{x}=\boldsymbol{A s}
$$

where $\boldsymbol{x}=\left(x_{1}, x_{2}, \cdots, x_{m}\right)^{\mathrm{T}}$ denotes the $m$-dimensional mixed signals which can be observed; $\boldsymbol{s}=\left(s_{1}, s_{2}, \cdots, s_{n}\right)^{\mathrm{T}}$ denotes the $n$-dimensional source signals; $\boldsymbol{A}$ is a constant $m \times n$ mixing matrix.

The target of BSS algorithm is to estimate the separating matrix, which satisfies the following equations:

$$
\boldsymbol{y}=\left(y_{1}, y_{2}, \cdots, y_{n}\right)^{\mathrm{T}}=\boldsymbol{W} \boldsymbol{x}
$$

where $\boldsymbol{y}$ is the $n$-dimensional separated signals, that is, the estimation of source signals.

Relying solely on the assumption of statistical independence of source signals, it is impossible to identify the mixed matrix completely or recover the source signals completely, so there are two ambiguities in the blind source separation method: order ambiguities and scale ambiguities of the separated signals (Hyvärinen, 2012).

\subsection{New Contrast Function}

Blind source separation is actually an optimization problem, and the optimization goal is the maximization of nonGaussianity. Kurtosis or its absolute value has been widely used in BSS and related fields as a measure of nonGaussianity, called contrast function, mainly for its simplicity in both computation and theory. This paper will take kurtosis as an example to describe the algorithm. In practical applications, we can also use other contrast functions such as negative entropy and mutual information. The kurtosis can be defined as:

$$
\operatorname{kurt}(y)=\mathrm{E}\left\{y^{4}\right\}-3\left(\mathrm{E}\left\{y^{2}\right\}\right)^{2}
$$

It should be noted that the variable $y$ in Eq. (4) is zero mean. To simplify the problem, it can be further assumed that $y$ has been normalized, that is, $\mathrm{E}\left\{y^{2}\right\}=1$ (Naik \& Kumar, 2011). Then the contrast function of the absolute value of the kurtosis can be simplified as:

$$
J(y)=|\operatorname{kurt}(y)|=\left|\mathrm{E}\left\{y^{4}\right\}-3\right|
$$

According to the known prior information, we construct the indexes that can reflect the morphological characteristics of the source signals, such as symmetry, stability, periodicity, skewness and so on, which are then introduced into the kurtosis contrast function. Actually, the similarity between the separated signal and the reference signal used in the traditional constrained BSS algorithm can also be regarded as a special case of the morphological characteristic index. Then the contrast function Eq. (5) is modified to:

$$
\overline{J(y)}=|k u r t(y)|+\lambda_{1} \delta_{1}(y)+\lambda_{2} \delta_{2}(y)+\cdots
$$

where $\delta_{\mathrm{i}}(y)$ denotes the morphological characteristic indexes; $\lambda_{i}$ is a factor to adjust the impact of $\delta_{\mathrm{i}}(y)$ to the contrast function.

It should be noted that the optimization target is the maximization of the contrast function. Therefore, the morphological characteristic index of the source signal needs to meet the maximum requirement.

\subsection{Optimization Calculation by Genetic Simulated Annealing Algorithm}

After establishing the new contrast function, the optimization calculation can be carried out to find the separation matrix $W$ which maximize $J(y)$. Due to the lack of explicit function expression, it is difficult to deduce the iterative formula. In this paper, optimization calculation is carried out by genetic simulated annealing algorithm (GSAA) (Wang, Wong \& Rahman, 2005), which combines the advantages of genetic algorithm and simulated annealing algorithm.

The result of algorithm operation is one row of the separating matrix $\boldsymbol{w}_{p}$, which satisfies:

$$
y_{p}=\boldsymbol{w}_{p} \boldsymbol{x}=\left(w_{p 1}, \cdots, w_{p m}\right) \boldsymbol{x}
$$

Each element $w_{p 1}, \cdots, w_{p m}$ in vector $\boldsymbol{w}_{p}$ is the variable to be calculated by GSAA, so the number of variables needed to be calculated is the number of mixed signals $m$. Because of 
the scale ambiguities, $w_{p 1}$ can be set to 1 , reducing the number of variables to $m-1$.

The fitness function of GSAA is the new contrast function Eq. (6) introduced in section 2.2:

$$
F_{f i t}=\overline{J(y)}=|\operatorname{kurt}(y)|+\lambda_{1} \delta_{1}(y)+\lambda_{2} \delta_{2}(y)+\cdots
$$

After obtaining the $\boldsymbol{w}_{p}$, the separated signal $y_{p}$ is obtained through Eq. (7).

\subsection{Extraction of Multiple Source Signals by Clustering}

One separated signal can be obtained every time the GSAA runs. To guarantee the separated signal to extract is different from the ones before, the Gram-Schmidt method is usually used to conduct deflationary orthogonalization so that the separated sources are uncorrelated to each other (Hyvarinen, 1999). However, in the mechanical system, the independence between the source signals is difficult to guarantee, which leads to the orthogonalization method no longer applicable. Therefore, a method of extracting multiple source signals by clustering is proposed.

The process of the method is as follows. Firstly, running GSAA repeatedly, all the source signals can be separated as long as the number of running times is enough. Then, all the separated source signals are clustered so that the separated signals corresponding to the same source can be classified into one cluster. Finally, the optimal separated signal is selected from each cluster respectively as the optimal estimation of each source signal.

In this paper, clustering is carried out by agglomerative clustering with average-linkage criterion (Himberg, Hyvärinen \& Esposito, 2004). The similarity between separated signals is usually measured by the absolute value of cross correlation coefficient $r_{i j}$. In fact, each correlation coefficient can be directly obtained by matrix operation:

$$
\boldsymbol{R}=\hat{\boldsymbol{W}} \boldsymbol{C} \hat{\boldsymbol{W}}
$$

where the element in the $i$ th row and jth column of matrix $R$ is the cross correlation coefficient between the $i$ th separated signal and the $j$ th separated signal, that is $r_{i j} ; \boldsymbol{C}$ is the covariance matrix of the mixed signals ; $\hat{\boldsymbol{W}}=\left[\hat{\boldsymbol{W}}_{1}^{T} \hat{\boldsymbol{W}}_{2}^{T} \cdots \hat{\boldsymbol{W}}_{M}^{T}\right]^{T}$ is the matrix formed by the separating vector $\hat{W}_{i}$, from each run $i=1,2, \cdots M$.

The final similarity matrix has the elements $\sigma_{i j}$ defined as:

$$
\sigma_{i j}=\left|r_{i j}\right|
$$

Dissimilarity index is needed in clustering methods. Therefore, it is necessary to transform the similarity matrix into a dissimilarity matrix with elements $d_{i j}$. A common transformation is given by:

$$
d_{i j}=1-\sigma_{i j}
$$

The cluster quality index is defined as:

$$
I_{R}=\frac{1}{L} \sum_{m=1}^{L} \frac{S_{m}^{i n}}{S_{m}^{e x}}
$$

where

$$
\begin{gathered}
S_{m}^{i n}=\frac{1}{\left|C_{m}\right|^{2}} \sum_{i, j \in C_{m}} d_{i j} \\
S_{m}^{e x}=\min _{m^{\prime} \neq m} \frac{1}{\left|C_{m}\right|\left|C^{\prime}{ }_{m}\right|} \sum_{i \in C_{m}} \sum_{j \in C_{m}^{\prime}} d_{i j}
\end{gathered}
$$

where $C_{m}$ denotes the set of indices that belong to the $m$ th cluster; $\left|C_{m}\right|$ denotes the size of the $m$ th cluster; $C_{m}^{\prime}$ denotes the set of indices that do not belong to the $m$ th cluster; $\left|C_{m}^{\prime}\right|$ denotes its size; $L$ denotes the number of clusters. $S_{m}^{i n}$ reflects the tightness within a cluster while $S_{m}^{e x}$ reflects the tightness between different clusters. Therefore, the index $I_{R}$ is an effective reflection of clustering tightness and the minimum of $I_{R}$ suggests the best partition.

The separated signals corresponding to the same source signal are clustered into one cluster. One with the largest contrast function value calculated by Eq. (6) can be considered as the optimal separated signal of each cluster. Through this process, all source signals can be extracted.

\subsection{Algorithm Steps}

To sum up, the steps of constrained blind source separation by morphological characteristics are as follows:

1. Set the parameters of GSAA.

2. Choose $M$, the number of times GSAA to run. Set $i=1$.

3. Run GSAA introduced in section 2.3 to obtain the separating vector $\hat{\boldsymbol{W}}_{i}$.

4. Set $i=i+1$. if $i$ is not greater than $M$, go back to step 3 .

5. The separating vector $\hat{W}_{i}$ from each run $i=1,2, \cdots M$ are collected into a single matrix $\hat{\boldsymbol{W}}=\left[\hat{\boldsymbol{W}}_{1}^{T} \hat{\boldsymbol{W}}_{2}^{T} \cdots \hat{\boldsymbol{W}}_{M}^{T}\right]^{T}$.

6. Choose $L$, the number of clusters. Run the clustering method introduced in section 2.4 and cluster $\hat{\boldsymbol{W}}_{i}$ $i=1,2, \cdots M$ into $L$ clusters. Set $j=1$.

7. Calculate the contrast function value of each separating vector in $j$ th cluster. The separating vector with the largest contrast function value is set to $\boldsymbol{w}_{j}$. 
8. Set $j=j+1$. if $j$ is not greater than $L$, go back to step 7 .

9. The matrix $\boldsymbol{W}=\left[\boldsymbol{w}_{1}^{T} \boldsymbol{w}_{2}^{T} \cdots \boldsymbol{w}_{L}^{T}\right]^{T}$ is the final separating matrix and the source signals is finally obtained by $\boldsymbol{y}=\boldsymbol{W} \boldsymbol{x}$.

\section{Application in MOdal ANAlysis}

\subsection{BSS in Modal Analysis}

Modal analysis can be divided into two steps: decomposing the response signal into single modal responses and modal parameter identification by the single modal identification technique. BSS can be applied to the decomposition of single modal responses, which is the basis for accurate identification of modal parameters.

The vibration of a n-degree-of-freedom (n-DOF) structural system could be described by the following equation:

$$
\boldsymbol{M} \ddot{\boldsymbol{x}}+\boldsymbol{C} \dot{\boldsymbol{x}}+\boldsymbol{K} \boldsymbol{x}=\boldsymbol{F}(t)
$$

where $\boldsymbol{M}, \boldsymbol{C}$ and $\boldsymbol{K}$ denote the mass matrix, damping matrix and stiffness matrix respectively; $\boldsymbol{x}, \dot{\boldsymbol{x}}$ and $\ddot{\boldsymbol{x}}$ denote the displacement vector, velocity vector and acceleration vector respectively; $\boldsymbol{F}(t)$ denote the external load vector.

For general engineering structures with small damping, the displacement response can be expressed by:

$$
\boldsymbol{x}(t)=\sum_{i=1}^{n} \boldsymbol{\psi}_{i} q_{i}(t)
$$

where $\psi_{i}$ denote the $i$ th mode shape vector; $q_{i}$ denote the normal coordinates.

The response of a system under random excitation is usually difficult to describe by time function. However, under wideband random excitation, such as white noise excitation, an undamped or less damped system responds mainly at frequencies equal to its natural frequencies, and the normal coordinates can be approximated by harmonic functions modulated by slowly varying envelopes $e_{i}(\mathrm{t})$ (Kerschen, Poncelet \& Golinval, 2007):

$$
\boldsymbol{x}(t) \cong \sum_{i=1}^{n} \boldsymbol{\varphi}_{i} e_{i}(t) \sin \left(\omega_{i} t+\phi_{i}\right)
$$

Set $q_{i}(t) \cong e_{i}(t) \sin \left(\omega_{i} t+\phi_{i}\right)$, which can be regarded as virtual source signals. It is not difficult to find that the Eq. (15) satisfies the mixing model of BSS problem.

In summary, under a random excitation, the modal decomposition of the responses of an n-DOF system can be regarded as a BSS problem.

\subsection{Simulation Experiment}

To verify the effectiveness of the algorithm, a simulation experiment is carried out in this section. A 3-DOF springmass vibration system is shown in Figure 1 , where $m_{1}=1$; $m_{2}=1 ; m_{3}=1 ; k=(100 \pi)^{2} ; k_{1}=2 k ; k_{2}=k ; k_{3}=k ; k_{4}=2 k ; k_{\mathrm{c}}=0.05$; $c 1=c 2=c 3=c 4=2 k_{\mathrm{c}} \times 100 \pi$; the random excitation $f(t)$ is applied to $m_{1}$.

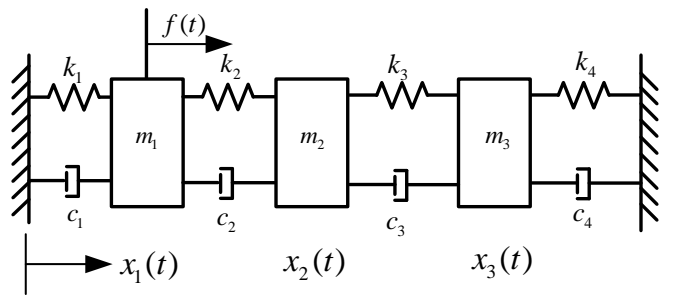

Figure 1. the 3-DOF spring-mass vibration system diagram.

Sampling frequency is $1000 \mathrm{~Hz}$. The displacement signals of the first 4 seconds are taken into account for the identification, as shown in Figure 2. The displacement signals are called the observed signals in the BSS problem, and also called the response signals in modal analysis.

(a)
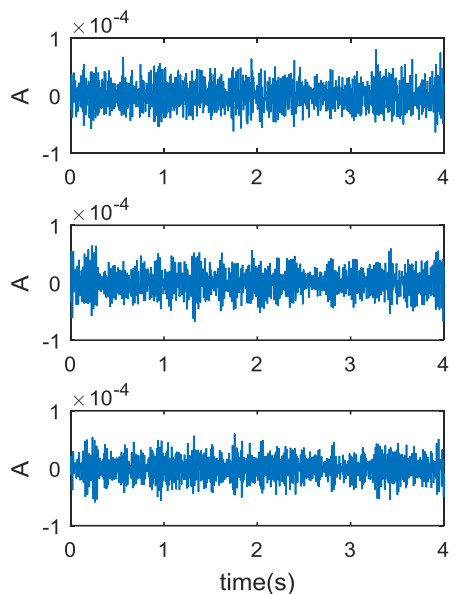

(b)
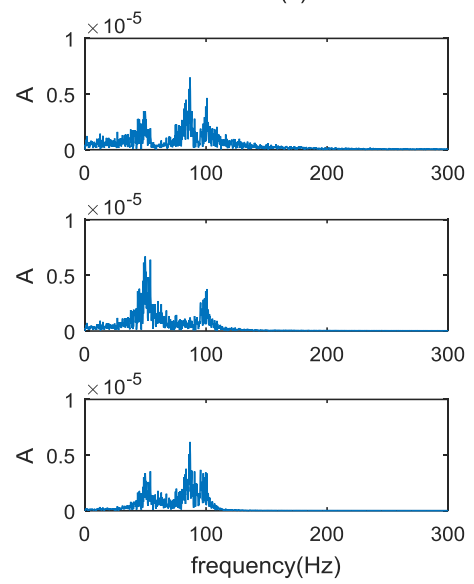

Figure 2. Response signals. (a) Waveforms of the observed signals. (b) Fourier spectrums of the observed signals. 
The fastICA algorithm is applied to the decomposition of the response signals. The waveform and spectrum of the separated signals $\boldsymbol{y}$ are shown in Figure 3.

(a)
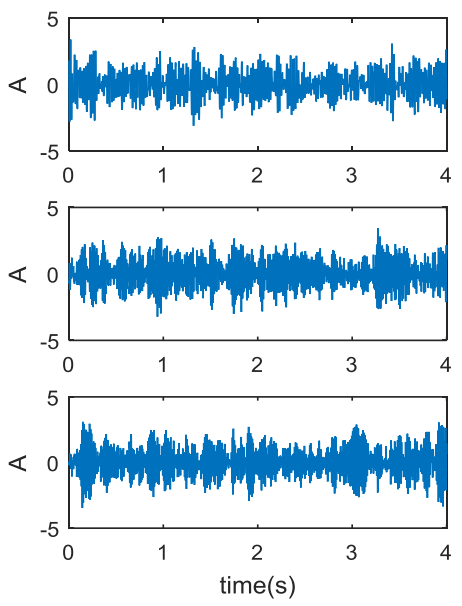

(b)
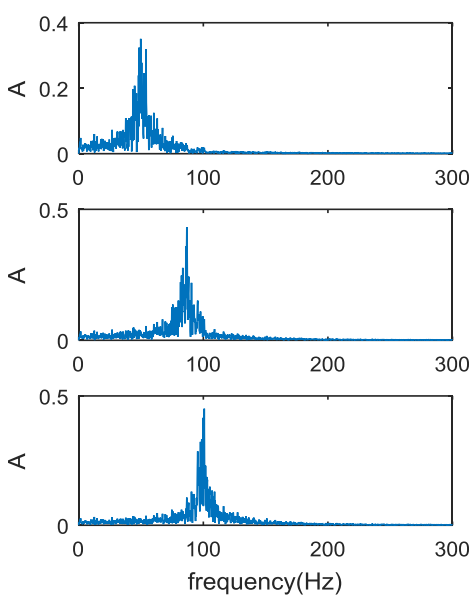

Figure 3. Results by the fastICA algorithm. (a) Waveforms of the separated signals $\boldsymbol{y}$. (b) Fourier spectrums of the separated signals $\boldsymbol{y}$.

In order to evaluate the effectiveness of the algorithm, it is necessary to compare the separated signals with the true single modal responses. In simulation, the true single modal responses $s$ can be obtained by calculation, as shown in Figure 4.

The similar index SNRs is used to evaluate the performances of the algorithms (Zhang, Zhang, Cheng, Li, Chen, Yang \& He, 2014):

$$
S N R s=10 \log _{10}\left(\sigma^{2} / \mathrm{MSE}\right)
$$

Where $\sigma^{2}$ denotes the variance of the true single modal responses $\boldsymbol{s}$; MSE denotes the mean square errors between the true single modal responses $\boldsymbol{s}$ and the separated signals $\boldsymbol{y}$. It should be noted that, it is necessary to transform the signals into equal variance before calculating the MSE due to the scale ambiguities. When the separated signal is similar to the source signal, the MSE between them is small. So the algorithm with bigger SNRs gets a better result.

(a)
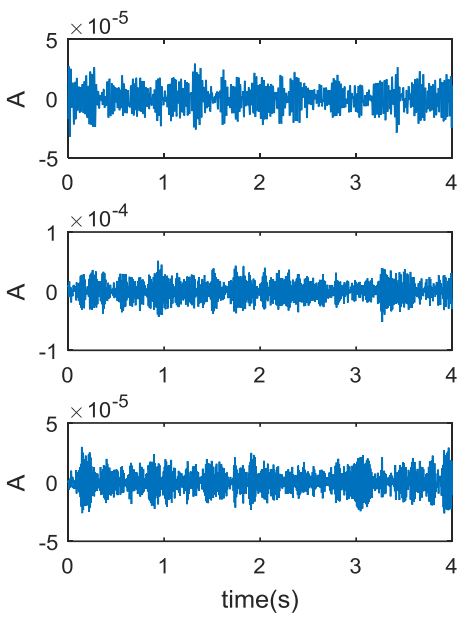

(b)
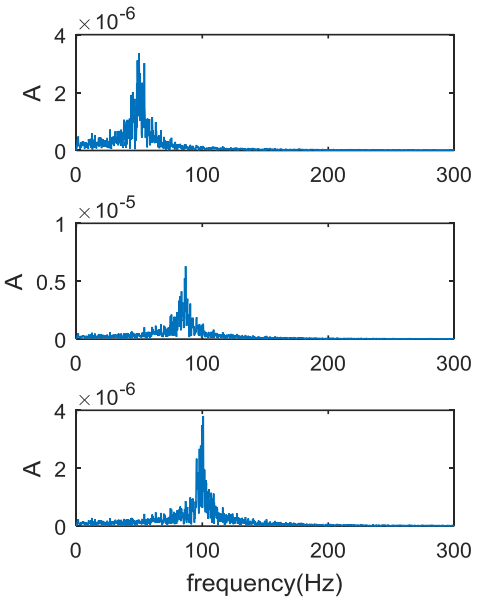

Figure 4. True single modal responses $\boldsymbol{s}$. (a) Waveforms of the single modal responses. (b) Fourier spectrums of the single modal responses.

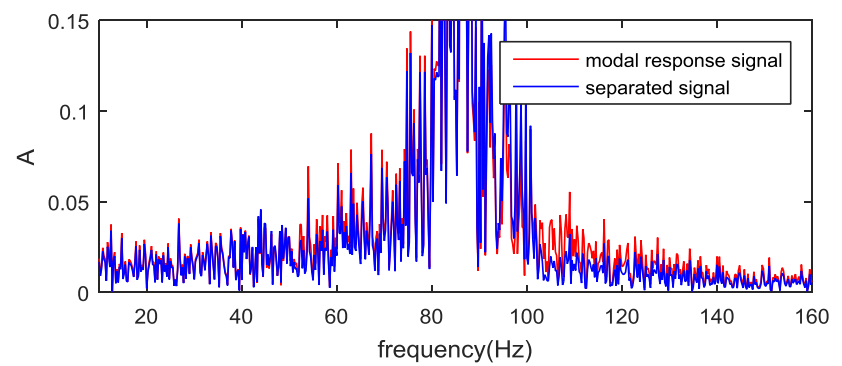

Figure 5. Comparison between the second order modal response signal and the second separated signal by the fastICA algorithm in Fourier spectrum.

The SNRs between each separated signal and each modal response signal is $24.07,14.25,25.77$, respectively. The first and third order modal response signals are accurately extracted, while the extraction of the second one is not accurate enough. Comparing the second separated signal and the second order modal response signal, we can see that 
the separated signal has obvious errors at $90 \sim 130 \mathrm{~Hz}$, as shown in Figure 5.

The independence assumption of BSS makes the separated signals uncorrelated to each other. The covariance matrix of separated signals $C_{y}$ is calculated:

$$
C_{y}=\left[\begin{array}{lll}
1 & 0 & 0 \\
0 & 1 & 0 \\
0 & 0 & 1
\end{array}\right]
$$

However, the true signal modal response signals are not strictly uncorrelated. The covariance matrix of true signal modal response signals $C_{s}$ is calculated:

$$
C_{s}=\left[\begin{array}{ccc}
1 & -0.0105 & 0.0086 \\
-0.0105 & 1 & -0.1969 \\
0.0086 & -0.1969 & 1
\end{array}\right]
$$

Therefore, it is not applicable to use independence criterion as separation target in this case. That is the reason why the result by the classical BSS algorithm is inaccurate.

Then, we use the algorithm proposed in this paper to decompose the responses signals into single modal responses.

It can be seen that the modal response signal of each order is approximately symmetrical about the peak in the Fourier spectrum, so the spectrum symmetry index can be used as the morphological characteristic index of the problem. To construct the index, the time-domain signal is transformed into its frequency-domain signal firstly. Then taking the peak frequency as symmetric center, the signal is split into two parts, the normalized correlation coefficient of which can be taken as the spectrum symmetry index.

$$
\delta(y)=\frac{\sum_{k=1}^{M}\left|\boldsymbol{Y}_{1}(k) \| \boldsymbol{Y}_{2}^{f}(k)\right|}{\left\|\boldsymbol{Y}_{1}(k)\right\|_{2}\left\|\boldsymbol{Y}_{2}^{f}(k)\right\|_{2}}
$$

where $\boldsymbol{Y}_{1}$ and $\boldsymbol{Y}_{2}$ are the two parts split into with sequence $M$ as the center; $\boldsymbol{Y}_{2}^{f}$ is the reverse of $\boldsymbol{Y}_{2} ;|\cdot|$ denotes the modulus of a vector; $\|\bullet\|_{2}$ denotes 2-norm. The value of the index is in the range of $[0,1]$. The larger the index, the stronger the spectrum symmetry of the signal.

The contrast function is obtained by taking Eq. (19) into Eq. (6). The combination of the kurtosis and the symmetry index makes the separated signals tend to be non-Gauss and symmetric.

Then, we can extract the single modal response signals from the observed signals according to the steps introduced in the section 2.5. The algorithm parameters are the scale $\lambda=2.5$, the number of times GSAA to run $M=30$, the number of clusters $L=3$. The separated signals is shown in Figure 6 .

(a)
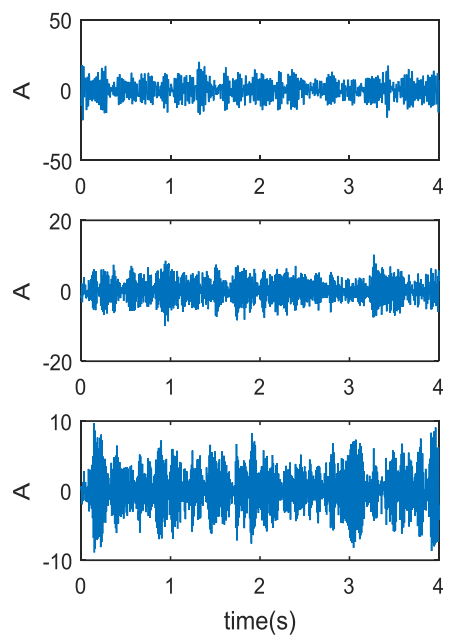

(b)
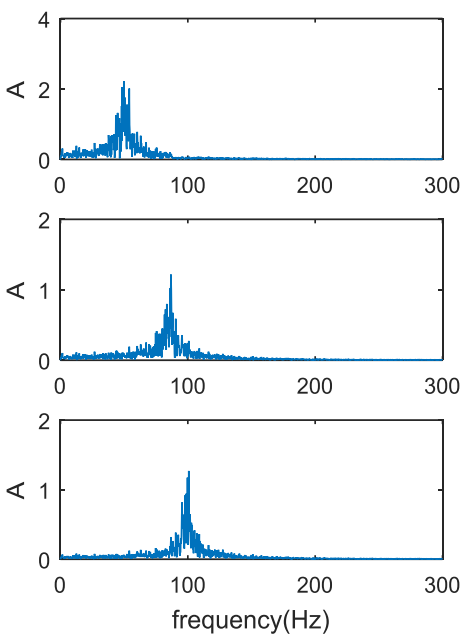

Figure 6. Results by the proposed algorithm. (a) Waveforms of the separated signals. (b) Fourier spectrums of the separated signals.

The SNRs between each separated signal and each modal response signal is $25.43,31.09,28.15$, respectively. The separation results of both fastICA algorithm and proposed algorithm are listed in Table 1. From the table, we can find that the first and third order modal response signals are still accurately extracted, while the extraction accuracy of the second one is significantly improved.

Table 1. Separation results.

\begin{tabular}{|l|l|l|l|}
\hline \multirow{2}{*}{ Method } & \multicolumn{3}{|l|}{ SNRs } \\
\cline { 2 - 4 } & Signal1 & Signal2 & Signal3 \\
\hline FastICA & 24.07 & 14.25 & 25.77 \\
\hline Proposed algorithm & 25.43 & 31.09 & 28.15 \\
\hline
\end{tabular}


Comparing the second separated signal and the second order modal response signal, we can see that the separated signal is basically coincided with the true modal response signal, as shown in Figure 7. It can be seen that the proposed constrained blind source separation by morphological characteristics has a better separation performance.

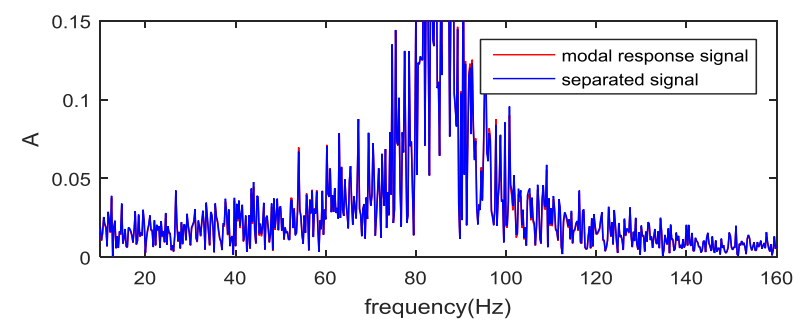

Figure 7. Comparison between the second order modal response signal and the second separated signal by the proposed algorithm in Fourier spectrum.

\section{Conclusion}

This paper proposes constrained BSS by morphological characteristics. The morphological characteristics indexes are constructed according to the prior information and then introduced into the BSS algorithm as a constraint to enhance the separation performance of the algorithm. The algorithm does not need to construct complete sequences of reference signals, so only a small amount of prior information is needed, which further extends the use of the prior knowledge in constrained BSS algorithm.

The proposed algorithm is applied to modal analysis of a 3DOF spring-mass vibration system under random excitation. The single modal response signals is extracted accurately from the response signals. By comparison, we can see that the accuracy of extraction has been significantly improved, verifying the superiority of the algorithm.

\section{ACKNOWLEDGEMENT}

This work was supported by National Natural Science Foundation of China (51775410), Science Challenge Project (TZ2018007).

\section{REFERENCES}

Benkedjouh, T., Zerhouni, N., \& Rechak, S. (2018). Tool wear condition monitoring based on continuous wavelet transform and blind source separation. International Journal of Advanced Manufacturing Technology, 97, 3311-3323. doi: 10.1007/s00170-018-2018-6.

Chang, J., Liu, W. B., Hu, H., \& Nagarajaiah, S.(2016). Improved independent component analysis based modal identification of higher damping structures. Measurement, $\quad 88, \quad 402-416$. doi:10.1016/j.measurement.2016.03.021.
He, J., Chen, Y., Zhang, Q. H., Sun, G. X., \& Hu, Q. (2018). Blind Source Separation Method for Bearing Vibration Signals. IEEE Access, 6, 658-664. doi:10.1109/ACCESS.2017.2773665.

Himberg, J., Hyvärinen, A., \& Esposito, F. (2004). Validating the independent components of neuroimaging time series via clustering and visualization. Neuroimage, 22(3), 1214-1222. doi:10.1016/j.neuroimage.2004.03.027.

Hyvarinen, A. (1999). Fast and robust fixed-point algorithms for independent component analysis. IEEE Transactions on Neural Networks, 10(3), 626-634. doi:10.1109/72.761722.

Hyvärinen, A. (2012). Independent component analysis: recent advances. Philosophical Transactions, 371(1984), 20110534. doi:10.1098/rsta.2011.0534.

Kerschen, G., Poncelet, F., \& Golinval, J. C. (2007). Physical interpretation of independent component analysis in structural dynamics. Mechanical Systems and Signal Processing, 21(4), 1561-1575. doi:10.1016/j.ymssp.2006.07.009.

Naik, G. R., \& Kumar, D. K. (2011). An overview of independent component analysis and its applications. Informatica, 35(1), 63-81.

Sadhu, A., Narasimhan, S., \& Antoni, J. (2017). A review of output-only structural mode identification literature employing blind source separation methods. Mechanical Systems and Signal Processing, 94, 415431. doi:10.1016/j.ymssp.2017.03.001.

Wang, Z. G., Wong, Y. S., \& Rahman, M. (2005). Development of a parallel optimization method based on genetic simulated annealing algorithm. Parallel Computing, $\quad 31(8), \quad 839-857$. doi:10.1016/j.parco.2005.03.006.

Zhang, J., Gao, H., Liu, Q., Farzadpour, F., Grebe, C., \& Tian, Y. (2017). Adaptive parameter blind source separation technique for wheel condition monitoring. Mechanical Systems and Signal Processing, 90, 208221. doi: 10.1016/j.ymssp.2016.12.021.

Zhang, J., Zhang, Z. S., Cheng, W., Li, X., Chen, B. Q., Yang, Z. B., \& He, Z. J. (2014). Kurtosis-Based Constrained Independent Component Analysis and Its Application on Source Contribution Quantitative Estimation. IEEE Transactions on Instrumentation \& Measurement, $\quad 63(7), \quad$ 1842-1854. doi:10.1109/TIM.2013.2293236.

Zhang, J., Zhang, Z. S., Cheng, W., Zhu, G., \& He, Z. (2015). A source contribution quantitative calculation method for mechanical systems based on the simplified independent component analysis with reference. Proceedings of the Institution of Mechanical Engineers Part C-Journal of Mechanical Engineering Science, 230(18), 3222-3240. doi: 10.1177/0954406215610788.

Zhao, Y., Kong, X., Jiang, H., \& Qu, M. (2014). Constrained independent component analysis techniques. In Proceedings-2014 IEEE Workshop on 
Electronics, Computer and Applications (419-422), May 8-9, Ottawa, ON, Canada. doi:10.1109/IWECA.2014.6845646.

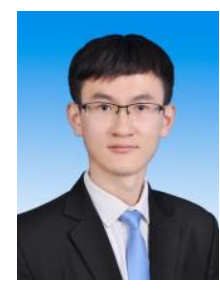

\section{BIOGRAPHIES}

Teng Gong was born in Laiyang, China, in 1990. He received the B.S. degree in mechanical engineering from Xi'an Jiaotong University, Xi'an, China, in 2013. $\mathrm{He}$ is currently pursuing the Ph.D. degree in mechanical engineering with the State Key Laboratory for Manufacturing Systems Engineering, Xi'an Jiaotong University, Xi'an, China. His research interests include blind source separation, intelligent diagnosis and condition monitoring.

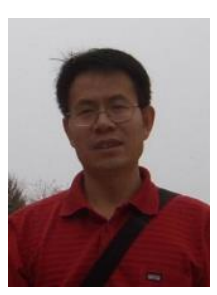

Zhousuo Zhang was born in Fengxiang, China, in 1964. He received the M.S. and Ph.D. degrees from Xi'an Jiaotong University, Xi'an, China, in 1991 and 2004, respectively. $\mathrm{He}$ is a Professor of mechanical engineering with Xi'an Jiaotong University. He was a Visiting Scholar with the University of Southampton, Southampton, U.K., from 2006 to 2007. His current research interests include condition monitoring, fault diagnosis and life prediction of mechanical equipment. 